\title{
Identification of some coffee leaf taxa using fluorescence spectroscopy and chemometrics
}

\author{
Saowaluk Madkoksung ${ }^{\mathrm{a}}$, Plaipol Dedvisitsakul ${ }^{\mathrm{a}, \mathrm{b}}$, Kanchana Watla-iad ${ }^{\mathrm{a}, \mathrm{c}, \mathrm{d}, *}$ \\ a School of Science, Mae Fah Luang University, Chiang Rai 57100 Thailand \\ b Microbial Products and Innovation Research Unit, School of Science, Mae Fah Luang University, \\ Chiang Rai 57100 Thailand \\ c Center of Chemical Innovation for Sustainability, School of Science, Mae Fah Luang University, \\ Chiang Rai 57100 Thailand \\ d Tea and Coffee Institute of Mae Fah Luang University, Mae Fah Luang University, Chiang Rai 57100 \\ Thailand
}

*Corresponding author, e-mail: kanchana.wat@mfu.ac.th

Received 11 Nov 2020

Accepted 20 May 2021

\begin{abstract}
Analytical techniques for identification of coffee taxa are essential for plant breeding and quality control of products. Rapid technique for discrimination of coffee taxa based on the fluorescence signals from their leaf extracts was introduced. Five different coffee taxa: Coffea liberica; Coffea congensis; Coffea arabica var. Geisha, a spontaneous hybrid of C. Arabica and Coffea canephora (Hibrido de timor), a hybrid of Hibrido de timor, and C. arabica var. Cattura; were investigated based on their fluorescence signals. The individual taxa present different fluorescence spectra. The spectra obtained from the excitation wavelengths at 300, 330, 390, 420, and $450 \mathrm{~nm}$; and emission wavelengths in the range of 500-790 nm were selected for principal component analysis (PCA) and partial least squares-discriminant analysis (PLSDA). It was found that fluorescence signals with excitation wavelength at $300 \mathrm{~nm}$ had been successfully implemented for rapid clustering and identification of some coffee taxa. The PCA score plot presenting natural clustering of data obtained from the fluorescence spectra tended to agree with the data of chemical contents based on antioxidant activity, total phenolic content, and total flavonoid content. The $Q^{2}$ and $R^{2}$ calculated via leave-one-out cross-validation (LOOCV) of model obtained from processing of the PLS-DA were 0.6 and 0.8 , respectively. It means that the model has potential for the categorization of coffee taxa based on their leaf extracts without any chemical treatments.
\end{abstract}

KEYWORDS: fluorescence spectroscopy, coffee leaves, coffee taxa, taxonomic identification, chemometric analysis

\section{INTRODUCTION}

Coffee species and varieties are important factors that affect coffee qualities. The identification of coffee is very important for the exploitation in coffee plant breeding and quality control of coffee products as well as the development of taste and favor diversity. There are more than 120 varieties of coffee, but the two most popular are $C$. arabica, commonly known as Arabica, and C. canephora known as Robusta [1]. Many scientific reports on taxonomic analyses of coffee are available on $C$. arabica and C. canephora. However, there are limited information of other coffee species and their varieties in the scientific literature. More information on genetic diversity of coffee would be beneficial to the development of coffee taste and favor diversity in the future. The discrimination of coffee taxa has been studied using morphological-based techniques [2], molecular DNA analysis [3], and traditional laboratorybased chemical methods [4]. In addition, there are many chemical analysis techniques applied for the classification of plant extracts obtained from various varieties, such as high-performance liquid chromatography-diode array (HPLC-DAD) [5], HPLC-diode array detector-time-of-flight-mass spectrometry (HPLC-DAD-TOF-MS) [6], and nuclear magnetic resonance spectroscopy (NMR) [7]. These techniques could improve performance of discrimination and classification by coupling with chemometric analysis. However, these methods require laborious and time consuming analytical workflow. Several spectroscopic techniques are successfully applied for taxonomic identification of living things due to their rapidity, cost effectiveness, and nontedious sample preparation $[8,9]$. Recently, the 
combinations of FT-NIR spectroscopy and chemometrics, such SIMCA analysis, have been successfully used to identify coffee leaves taxa [10]. Besides, Laser-Induced Breakdown Spectroscopy and chemometrics have been used to identify coffee varieties from their beans [11]. Fluorescence spectroscopy is one of the rapid techniques implemented for taxonomic study in a wide range of organisms such as bacteria [12-15], microalgae [16], fungi, and plants [17-19]. Due to the presence of various autofluorescent molecules, such as nicotinamides (NADPH, NAD), pterins, phenols (hydroxycinnamic acid), alkaloids, flavins (FAD, FMN), flavonoids, terpenoids, polyacetylenes, isoquinolines, chlorophylls, anthocyanins, and anthocyanidins [20], leaves of typical green plants provide numerous differences of fluorescence spectra. Therefore, fluorescence spectra from leaves were widely used for fingerprint construction and taxonomic identification of plants. There are abundances of various fluorescent secondary metabolites in coffee leaves, such as chlorogenic acid (5caffeoyl-quinic acid, 5-CQA)), mangiferin (C2- $\beta$-Dglucoside-1,3,6,7-tetrahydroxyxanthen-9-one), and caffeine (1,3,7-trimethylxanthine) [20]. However, the literature contains no report on the use of fluorescence spectroscopy for coffee taxonomic study. Identification of coffee taxa based on their leaves is interesting because leaves could be collected easily all year round and no limitation of fruiting season.

In the present study, cost effective, rapid, and non-destructive fluorescence spectroscopy combined with chemometrics, such as principal component analysis (PCA) and partial least squares discriminant analysis (PLS-DA), were used for the identification of some coffee leaf taxa obtained from the same planting area [21]. The algorithm of PCA, an unsupervised method, was processed to understand the natural clustering of the fluorescence signals of coffee leaf extract without referring to class labels. Because coffee leaf contains various fluorescent secondary metabolites presenting biological activities, such as chlorogenic acids (CGA) and mangiferin [20,22], the PCA results obtained from fluorescence spectra were compared with the PCA data from antioxidant activity, total phenolic content, and total flavonoid content of coffee leaf samples. Hierarchical cluster analysis (HCA) in the form of dendrogram was also used to demonstrate the interrelationships between samples. Then, the algorithm of PLS-DA, a supervised method, was performed to construct a model for classification of coffee taxa.

\section{MATERIALS AND METHODS}

\section{Chemicals and reagents}

Methanol and sodium hydroxide (97\%) were purchased from RCI Labscan Company (Thailand). The $\mathrm{L}(+)$-ascorbic acid standard was obtained from POCH (Poland). The 2,2-diphenyl-1-picrylhydrazyl $(\mathrm{DPPH})$ and the $( \pm)$-catechin hydrate primary reference standard was purchased from Sigma Aldrich (US). The gallic acid monohydrate standard was purchased from Fluka (Spain). Sodium carbonate anhydrous was obtained from Ajax Finechem (Australia) and aluminium chloride hexahydrate (AR Grade) was ordered from QReC (New Zealand). Sodium nitrite (98\%) was obtained from LOBA CHEMIE (India). Folin-Ciocaltue's phenol reagent was purchased from MERK (Germany). All reagents and chemicals used were of analytical grade.

\section{Sampling and sample preparations}

The coffee leaves of four different coffee taxa (C. liberica, C. arabica var. Geisha, a spontaneous hybrid of $C$. arabica and C. canephora (Hibrido de timor) named Hibrido de timor hereafter, and a hybrid of Hibrido de timor and C. arabica var. Cattura (named H306/1 ML1/1 hereafter) were collected for chemical content analyses (antioxidant activity, total phenolic content, and total flavonoid content). The same four taxa plus $C$. congensis were collected for fluorescence analysis. Those coffee leaves were obtained from Mae Lod Royal Agricultural Research Station (the Royal Project Foundation), Chiang Mai, Thailand in mid-December (during fruit ripening period) 2018 and 2019.

The sampling of coffee leaves was carried out using the minor modified method of Sousa et al [23] and Tamimi et al [24]. The five branches were selected by counting down from the top of the vertical to the 8 th to 12 th lateral branch. Then, the recently matured leaves from these laterals, usually the 3rd or 4th pair back from the branch tip, were picked. These leaves should be full-sized with the same color and texture. Three coffee trees of each taxon were marked, and at least 15 leaves were collected per tree.

All leaf samples were cleaned, dried at $60^{\circ} \mathrm{C}$ in an oven for $3 \mathrm{~h}$, and then ground into powder using a blender. The extraction was performed in triplicate by soaking $0.1 \mathrm{~g}$ coffee leaf powder in $1.5 \mathrm{ml}$ of methanol for $12 \mathrm{~h}$ followed by shaking the sample solutions for $3 \mathrm{~h}$ at $150 \mathrm{rpm}$ in a water bath shaker (Wisd laboratory instrument, Korea). Then the solutions were filtered into $5 \mathrm{ml}$-volumetric 
flasks, adjusted the volume to $5 \mathrm{ml}$, and stored in a refrigerator for further analyses.

\section{Antioxidant activity assay}

The DPPH radical scavenging activity of the methanolic extracts obtained from coffee leaf samples were carried out using the minor modified method of Saw et al [25]. Solution of 2,2-diphenyl1-picrylhydrazyl (DPPH, $0.3 \mathrm{mM}$ ) was prepared in methanol. Aliquots of $200 \mu \mathrm{l}$ of sample extracts were mixed with $1 \mathrm{ml}$ of DPPH solution. Then, the solution's volume was adjusted to $4 \mathrm{ml}$ by ultrapure water (Millipore Milli-Q ${ }^{\mathrm{TM}}$ Reference Ultrapure Water Purification System), mixed by a vortex mixer (VM-10 model, Wisd laboratory instrument, Korea), and then incubated for $30 \mathrm{~min}$ in a dark room. Standard solutions of ascorbic acid in the concentration range of $0-0.018 \mathrm{mM}$ were prepared. The absorbance of the solutions was measured at $515 \mathrm{~nm}$ [26] using a visible spectrophotometer (USB4000, Ocean Optics, USA). The DPPH radical scavenging activities of all extracts were calculated using the ascorbic standard curve. The results were presented as milligram ascorbic acid equivalent per gram dried coffee leaf weight (mgAAE/g dried weight).

\section{Total phenolic content}

Analyses of the total phenolic content in coffee leaf extracts were carried out using the method proposed by Haile et al [27] with minor modification. Briefly, Folin-Ciocalteu reagent (10\% w/v) dissolved in methanol was used as reagent. The leaf extract sample $(150 \mu \mathrm{l})$ was mixed with the Folin-Ciocalteu reagent $(125 \mu \mathrm{l})$ and incubated for $5 \mathrm{~min}$. Then, the solution was mixed with $1.25 \mathrm{ml}$ of $\mathrm{Na}_{2} \mathrm{CO}_{3}$ $(7 \% \mathrm{w} / \mathrm{v})$ and adjusted the volume by using ultrapure water to $3 \mathrm{ml}$. The solution was mixed and then incubated in a dark room for $1 \mathrm{~h}$. After that, the absorbance of the solution was measured at $765 \mathrm{~nm}$ using a visible spectrophotometer. Different concentrations of gallic acid standard solution were prepared in the range of 0 to $100 \mathrm{ppm}$. The total phenolic content was presented as milligram gallic acid equivalent per gram dried coffee leaf weight (mgGAE/g dried weight).

\section{Total flavonoid content}

Analyses of total flavonoids content in coffee leaf extracts were carried out using the method proposed by Phuyal et al [28] with minor modification. The leaf extract sample $(200 \mu \mathrm{l})$ was mixed with $0.55 \mathrm{M}$ of $\mathrm{AlCl}_{3}(100 \mu \mathrm{l})$ and, then, $3.0 \mathrm{M}$ of $\mathrm{NaNO}_{2}$
$(50 \mu 1)$. After that, $2.5 \mathrm{M} \mathrm{NaOH}(250 \mu \mathrm{l})$ was added into the mixture solution. The solution volume was adjusted to $2 \mathrm{ml}$ by ultra-pure water. The solution was mixed and incubated for $30 \mathrm{~min}$ in a dark room. The absorbance of the solution was measured at $510 \mathrm{~nm}$ by using a visible spectrophotometer. The calibration standard curve was prepared using catechin standard in the concentration range of 0 to $100 \mathrm{ppm}$. The flavonoids content was presented as milligram catechin equivalent per gram dried weight (mgCE/g dried weight).

\section{Measurement of fluorescence spectra}

A fluorescence spectrophotometer (LS55 model, PerkinElmer, USA) equipped with a xenon lamp and a photomultiplier detector was used for fluorescence spectrum analysis of the leaf extract samples. The excitation wavelength for detecting fluorescence signal of the studied solution was set from 270 to $460 \mathrm{~nm}$. The emission wavelength was monitored in the range of 500 to $800 \mathrm{~nm}$. All the measurements were performed in a standard fluorescence 96-well plate with a scan rate at $800 \mathrm{~nm} / \mathrm{min}$ and $10 \mathrm{~nm}$ bandwidths for the emission and excitation monochromators.

\section{Statistical and multivariate analysis}

The spectra of 3D-fluorescence contour plot of all sample extracts were investigated for selecting the excitation wavelengths and the range of emission wavelength. The excitation wavelengths producing different fluorescence intensities in the range of 500-790 nm were selected for further statistical and multivariate analyses. Then, the data were filtered using interquartile range before processing. Normalization was performed by the sample median method. The fluorescence intensities obtained from the selected excitation wavelengths of all extracts were processed by a PCA to study the natural clustering of the samples. Statistical analyses were performed by mean of the MetaboAnalyst ${ }^{\circledR} 4.0$ online program running by R-script chemometrics [29]. The PCA clustering result obtained from the data set of fluorescence spectra was compared with that obtained from the data set of chemical contents based on antioxidant activity, total phenolic content, and total flavonoid content of coffee leave samples.

Then, hierarchical cluster analysis (HCA) in the form of a dendrogram was also used to study the interrelationships between samples. Two important parameters were considered for performing HCA. The first one was similarity measure including Euclidean distance, Pearson's correlation, and 
Spearman's rank correlation. The other parameter was clustering algorithms including average linkage, complete linkage, single linkage, and Ward's linkage.

Finally, partial least squares - discriminant analysis (PLS-DA) was processed to develop a model for classification of the membership. Leave-oneout cross validation (LOOCV) was used for cross validation. The quality assessments of $Q^{2}$ and $R^{2}$ calculated via cross-validation (CV) were used for the estimation of the qualitative measurement of the model consistency between the predicted and the original data [30]. In addition, Variable Importance in Projection (VIP) values obtained from sum of squares of the PLS loadings, which consider the amount of explained $Y$-variation in each dimension [31], were also calculated.

\section{RESULTS AND DISCUSSION}

\section{Multivariate analysis of antioxidant activity, total flavonoid content, and total phenolic content}

The chemical contents, including antioxidant activity, total flavonoid content, and total phenolic content, found in four coffee leaf extracts (C. liberica, C. arabica var. Geisha, Hibrido de timor, and hybrid H306/1 ML1/1) are shown in Fig. 1. The chemical contents found in individual coffee leaf taxa seem to be different. The 2D-scores plot between the selected principal components (PCs) obtained from the PCA using the chemical contents of the samples are shown in Fig. 2. The result showed that the C. liberica, C. arabica var. Geisha, and Hibrido de timor samples could be clustered clearly according to their chemical contents. The literature reported that the coffee beans of $C$. liberica presented higher chemical contents (based on the result of the total phenol assay, the DPPH assay, and the ferric reducing antioxidant power (FRAP) assay) than that of C. robusta and C. arabica [32]. However, the H306/1 ML1/1 (hybrid coffee taxon) could not be clustered clearly from the C. liberica and C. arabica var. Geisha. Therefore, the data set from other chemical factors might be required for classification of hybrid coffee taxa.

\section{Fluorescence spectral characteristics of coffee leave extracts}

Fluorescence spectra with emission spectra in horizontal axis and excitation spectra in vertical axis of the methanolic coffee leaf extracts (C. liberica, C. congensis, C. arabica var. Geisha, Hibrido de timor,

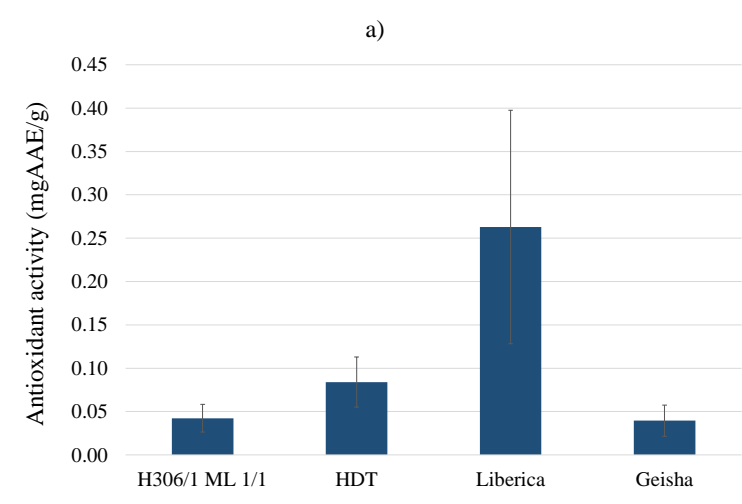

b)
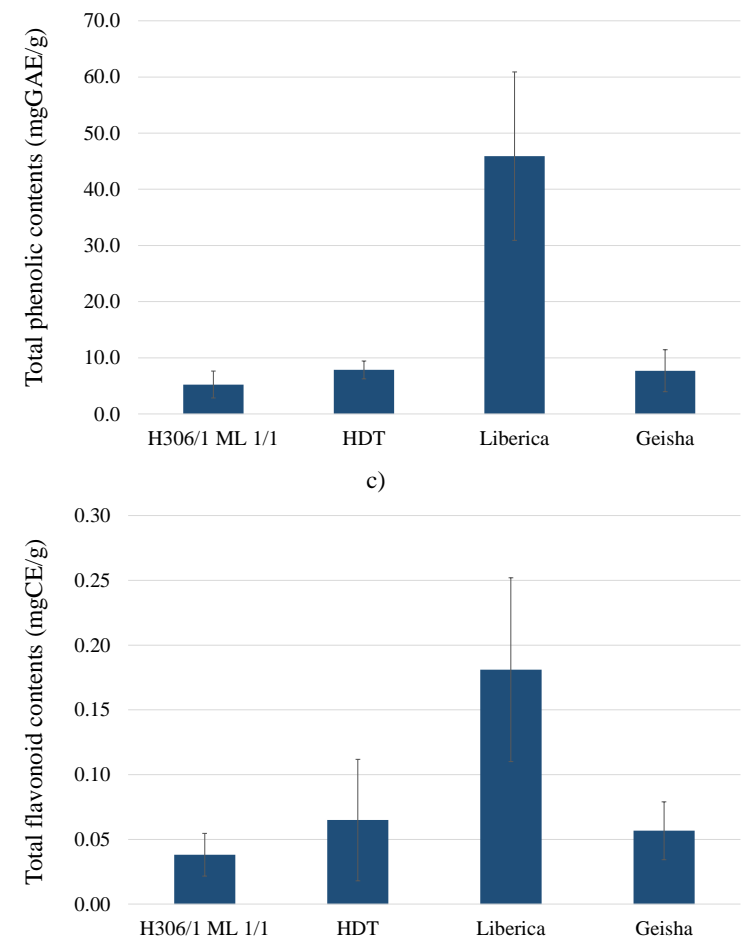

Fig. 1 Antioxidant activities (a), total phenolic content (b), and total flavonoid content (c) found in four coffee leaf extracts: C. liberica (Liberica), C. arabica var. Geisha (Geisha), Hibrido de timor (HDT), and a hybrid H306/1 ML1/1.

and H306/1 ML1/1) are shown in Fig. 3. The contour line patterns of each coffee leaf extract were different. These fluorescence results might be due to the differences of concentration of chemical composition in the extract samples as reported by Yisak et al [32]. Besides, it could be observed that excitation wavelength at $420 \mathrm{~nm}$ provided high intensity of fluorescence signal. The fluorescence intensities of each sample obtained from excitation 

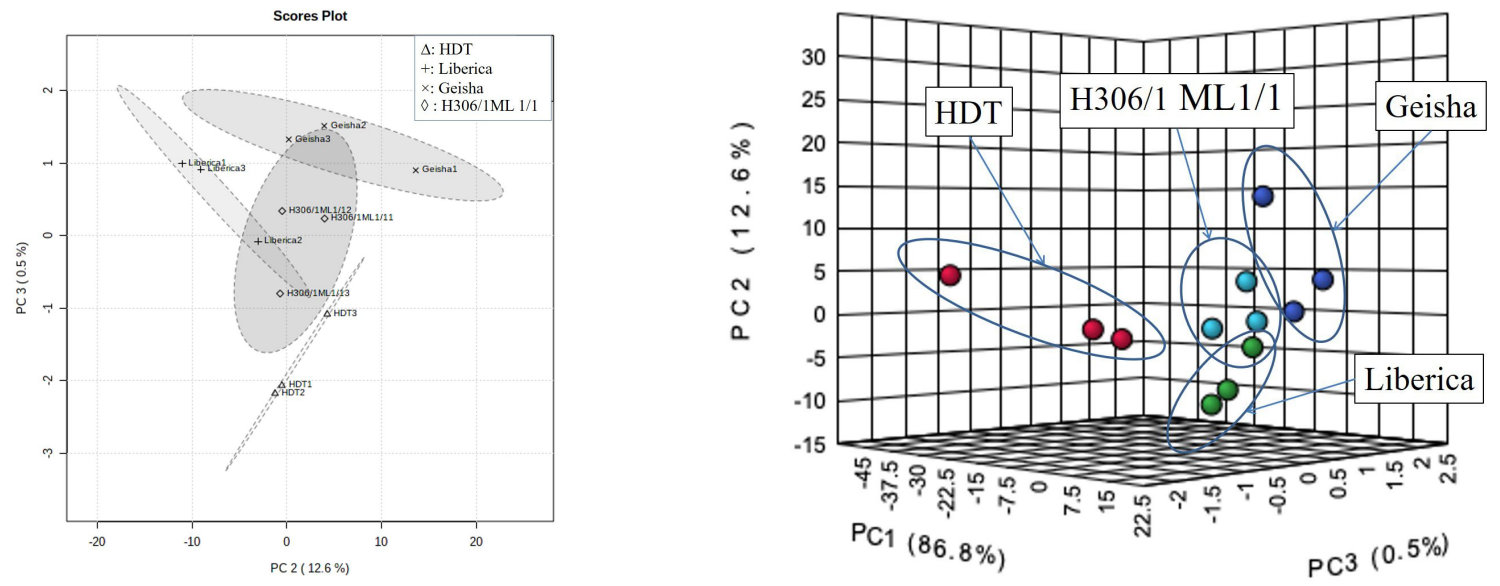

Fig. 2 The 2D (a) and 3D-scores plot (b) between the selected principal components (PCs) obtained from the principal component analysis (PCA) according to the chemical contents of the samples: C. liberica (+), C. arabica var. Geisha $(\times)$, Hibrido de timor $(\triangle)$, and hybrid H306/1 ML1/1 $(\diamond)$.
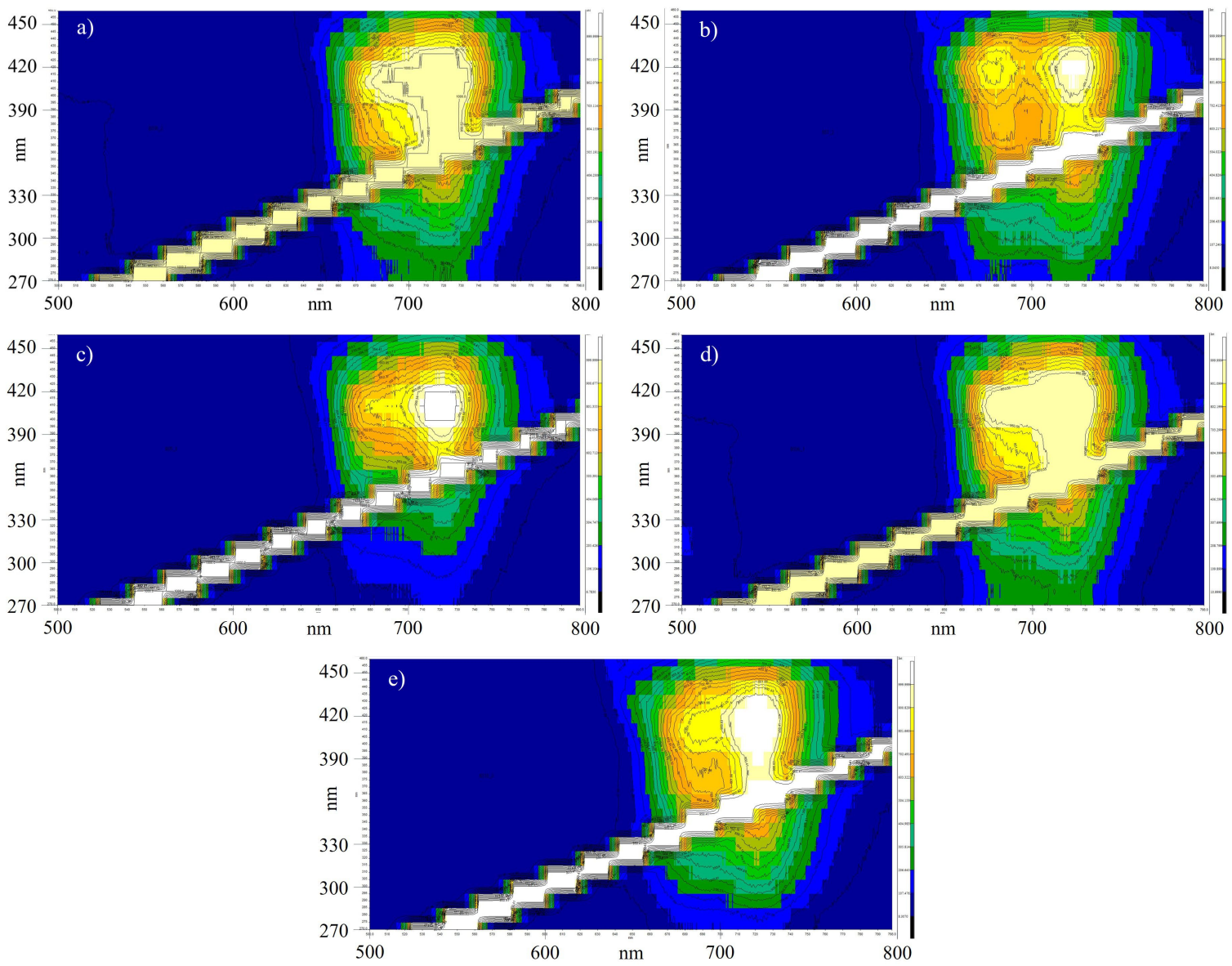

Fig. 3 Contour plots of the fluorescence spectra with emission spectra in horizontal axis and excitation spectra in vertical axis of the methanolic coffee leaf extracts: C. liberica (a), C. congensis (b), C. arabica var. Geisha (c), Hibrido de timor (d), and a hybrid H306/1 ML1/1 (e). 


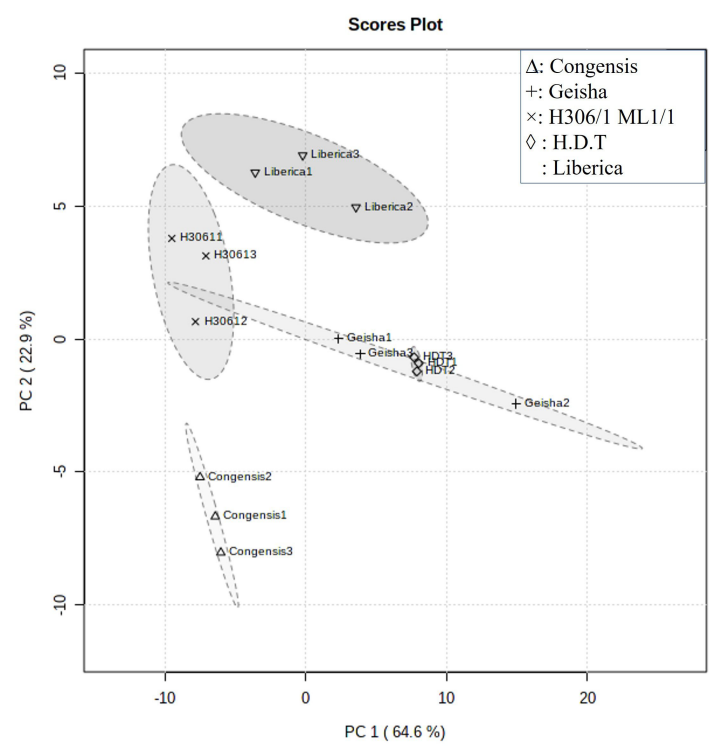

Fig. 4 The scores plot between the selected principal components $(\mathrm{PC} 1 \times \mathrm{PC} 2)$ of raw spectra obtained from using excitation wavelengths at $300 \mathrm{~nm}$ : $C$. liberica $(\nabla)$, C. congensis $(\triangle)$, C. arabica var. Geisha $(+)$, Hibrido de timor $(\diamond)$, and hybrid H306/1 ML1/1 $(\times)$.

wavelength at $300,330,390,420$, and $450 \mathrm{~nm}$ and the emission range from 500 to $790 \mathrm{~nm}$ were selected to perform multivariate analysis for distinguishing coffee taxa, based on the differences of fluorescence intensity.

\section{Multivariate analysis of fluorescence spectra}

A PCA of the selected fluorescence intensities was processed via the MetaboAnalyst ${ }^{\circledR} 4.0$ (web online) to examine the natural clustering of the coffee taxa. The fluorescence intensities obtained from the combination of emission wavelengths and excitation wavelengths were assigned as data sets. The scores plot between the selected PCs of individual spectra obtained from using several excitation wavelengths was considered. It was found that $C$. liberica, C. arabica var. Geisha, hybrid H306/1ML 1/1, and C. congensis could be clustered clearly using fluorescence intensity from the excitation wavelength at $300 \mathrm{~nm}$, except Hibrido de timor coffee that showed overlapping with $C$. arabica var. Geisha (Fig. 4). Using fluorescence spectra obtained from excitation wavelength at $300 \mathrm{~nm}$ tended to separate the data set as compared with the results of chemical contents, which could separate only three coffee taxa.

The model using fluorescence intensities ob- tained from excitation wavelength at $300 \mathrm{~nm}$ of individual coffee leaf taxa could be performed using partial least squares-discriminant analysis (PLSDA). Variable Importance in Projection (VIP) values obtained from sum of squares of the PLS loadings and performance of classification are shown in Fig. 5. The VIP values presented that the fluorescence intensities obtained from emission wavelengths from 580 to $620 \mathrm{~nm}$ had influence on classification of coffee taxa. The results might be affected by alkaloids found in coffee, such as caffeine, theobromine, and trigonelline, as their maximum UV-visible excitation bands were obtained at $272.89 \mathrm{~nm}, 272.73 \mathrm{~nm}$, and $264.59 \mathrm{~nm}$, respectively $[32,33]$. The amount of those alkaloids in green coffee beans is influenced by numerous factors such as coffee variety, genetic properties of the cultivars, and environment [34]. Moreover, secondary metabolites in coffee leaves that might influence the clustering result are chlorogenic acid (5-CQA) and mangiferin (phenolic compounds) because both compounds exhibit a broad spectral range with peaks at about 425 and $520 \mathrm{~nm}$, respectively. Other compounds of influence might be flavins, flavonoids, some terpenoids, polyacetylene, isoquinoline, and some alkaloids because these compounds emit fluorescence in the red region of the visible spectrum [20]. The quality assessments of $Q^{2}$ and $R^{2}$ calculated via Leave-one-out crossvalidation (LOOCV) were 0.6 and 0.8 , respectively (Fig. 5). The red star in Fig. 5 indicates the best classifier. The literature suggests that $R^{2}$ value of 0.67 is substantial model [35]. Although $Q^{2}$ has no standard of comparison or critical value for inferring significance, an empirically inferred value of $\geqslant 0.4$ is acceptable for a biological model [36]. The $Q^{2}$ is close to the $R^{2}$ when the PLS built on a training set is applied to a test set. It means that PLS model works independently of the specific data used to train the PLS model. Therefore, there is a tendency to use the fluorescence spectra of methanolic-coffee leaf extracts obtained from the excitation wavelength at $300 \mathrm{~nm}$, and the emission range from 500 to $790 \mathrm{~nm}$ for identification of coffee taxa.

An optimized dendrogram of raw fluorescence spectra of coffee leaf extracts (emission wavelength at $300 \mathrm{~nm}$ ), using Euclidean distance as similarity measure and average linkage as clustering algorithm is shown in Fig. 6. The groups of C. liberica, hybrid H306/1ML 1/1, and C. congensis were well separated. However, the groups of $C$. arabica var. Geisha and Hibrido de timor were overlapped. The natural clustering of the samples presented as a den- 

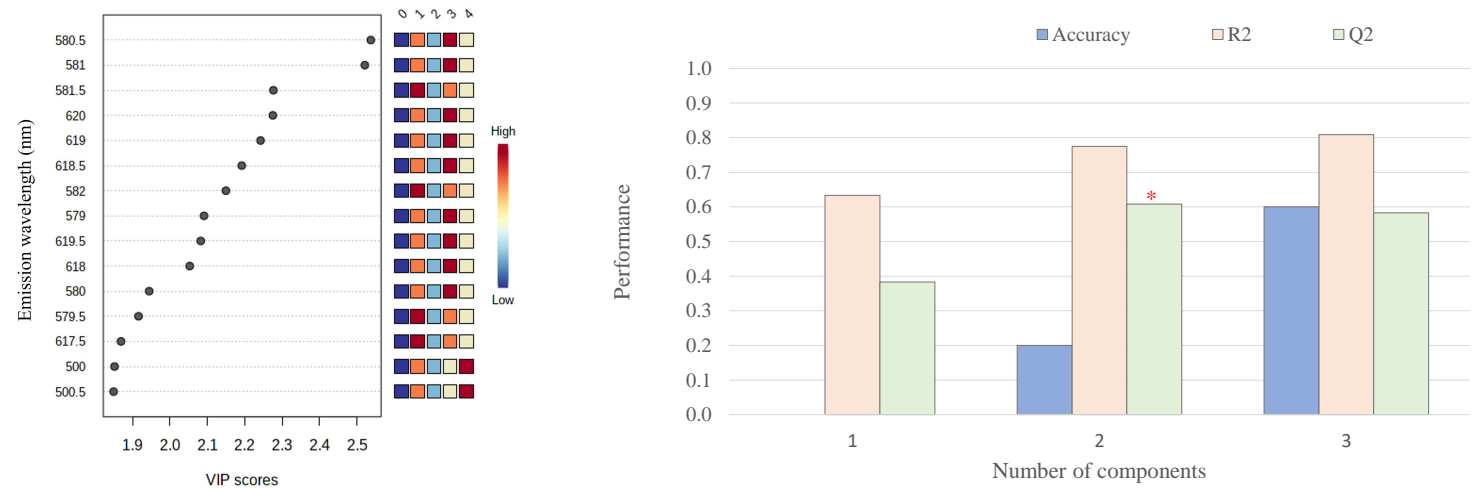

Fig. 5 Variable importance in projection (VIP) values obtained from sum of squares of the PLS loadings (a) and performance of classification; (b) C. congensis (group 0), C. arabica var. Geisha (group 1), hybrid H306/1 ML1/1 (group 2), Hibrido de timor (group 3), and C. liberica (group 4).

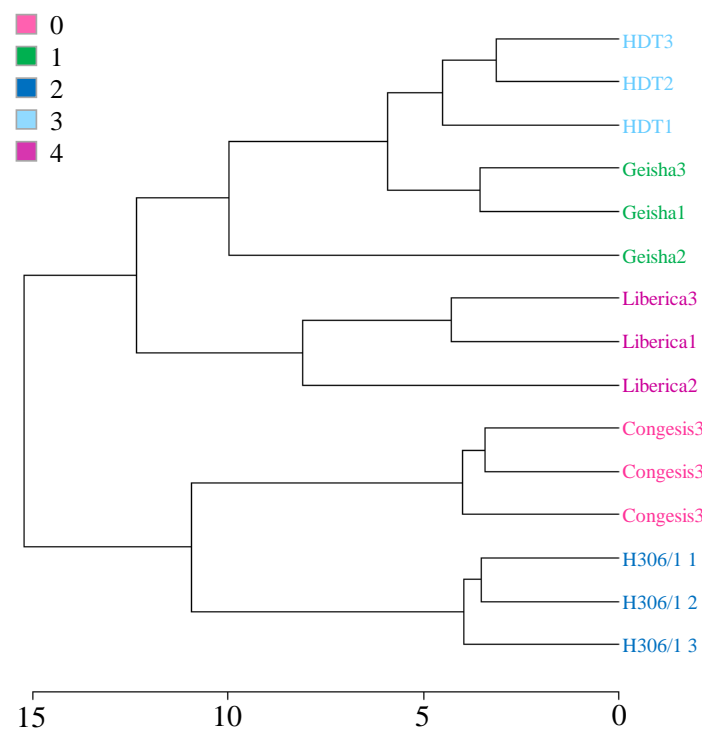

Fig. 6 Clustering result shown as dendrogram (distance measure using Euclidean and clustering algorithm using average): C. congensis (0), C. arabica var. Geisha (1), hybrid H306/1 ML1/1 (2), Hibrido de timor, HDT (3), and C. liberica (4).

drogram agrees with the PCA scores plot between the selected principal components $(\mathrm{PC} 1 \times \mathrm{PC} 2)$ of raw spectra obtained from excitation wavelength at $300 \mathrm{~nm}$. This result might be due to genetic factors. Both C. arabica var. Geisha and Hibrido de timor coffee originated from same Arabica variety.

Multivariate analysis of both the chemical contents and the fluorescence spectra obtained from coffee leaf extracts could be applied for study of the natural clustering in data. However, the flu- orescence spectroscopy coupled with chemometric techniques for data clustering and identification is preferred because the analytical methods are chemical and time consuming. On the other hand, the fluorescence technique requires only a few easy steps of sample extraction. In addition, fluorescence spectrum is represented from various autofluorescent metabolites in sample. It is proved to be simple, high sensitivity, and less sample consumption [37].

\section{CONCLUSION}

Multivariate analysis of the data of chemical contents and fluorescence spectra for simple classification of some coffee leave taxa extracted with methanol was studied. Principal component analysis according to chemical contents obtained from antioxidant activity, total flavonoid content, and total phenolic content could cluster clearly between Hibrido de timor, C. liberica and C. arabica var. Geisha. The PCA score plot of fluorescence spectra obtained from excitation wavelength at $300 \mathrm{~nm}$ also separated clearly between C. liberica, hybrid H306/1ML 1/1, and C. congensis. It agrees with the result of the hierarchical cluster analysis presented as a dendrogram. Due to the PLS model works independently on specific data, there is a tendency to use the fluorescence spectra of methanolic extracts obtained from the excitation wavelength at $300 \mathrm{~nm}$ and the emission range from 500 to $790 \mathrm{~nm}$ for coffee classification study. Fluorescence spectroscopy coupled with chemometric techniques is recommended as screening methods for coffee taxa identification because it requires a few easy steps of sample extraction. It is proved to be simple, high sensitivity, and less sample consumption. 
Acknowledgements: This work was financial supported by the National Research Council of Thailand, NRCT (17549). We would also thank the Mae Lod Royal Agricultural Research Station, Royal Project Foundation, Chiang Mai (Thailand) for coffee leaf samples and Mr. Jakkarach Sa-udon for coffee leaf sampling and fruitful discussion.

\section{REFERENCES}

1. Chamyuang S, Owatworakit A, Intatha U, Duangphet S (2021) Coffee pectin production: An alternative way for agricultural waste management in coffee farms. ScienceAsia 47S, 90-95.

2. Anthony F, Bertrand B, Quiros O, Wilches A, Lashermes P, Berthaud J, Charrier A (2001) Genetic diversity of wild coffee (Coffea arabica L.) using molecular markers. Euphytica 118, 53-65.

3. Mishra MK, Huded AKC, Jingade P (2020) Assessment of the suitability of molecular SCoT markers for genetic analysis of coffee species. Botanica 26, 184-196.

4. Martín MJ, Pablos F, González AG (1998) Discrimination between arabica and robusta green coffee varieties according to their chemical composition. Talanta 46, 1259-1264.

5. Japon-Lujan R, Ruiz-Jiménez J, Luque de Castro MD (2006) Discrimination and classification of olive tree varieties and cultivation zones by biophenol contents. J Agric Food Chem 54, 9706-9712.

6. Talhaoui N, Gómez-Caravaca AM, Roldan C, Leon L, De la Rosa R, Fernandez-Gutierrez A, SeguraCarretero A (2015) Chemometric analysis for the evaluation of phenolic patterns in olive leaves from six cultivars at different growth stages. $J$ Agric Food Chem 63, 1722-1729.

7. Amargianitaki M, Spyros A (2017) NMR-based metabolomics in wine quality control and authentication. Chem Bio Technol Agric 4, ID 9.

8. Kucharska-Ambrożej K, Karpinska J (2020) The application of spectroscopic techniques in combination with chemometrics for detection adulteration of some herbs and spices. Microchem J 153, ID 104278.

9. Khamchum C, Punsuvon V, Kasemsumran S, Suttiwijitpukdee N (2013) A feasibility study of oil content and fatty acid composition of seed powder and seed oil of Pongamia pinnata by near infrared spectroscopy. ScienceAsia 39, 384-391.

10. Mees C, Souard F, Delporte C, Deconinck E, Stoffelen P, Stévigny C, De Braekeleer K (2018) Identification of coffee leaves using FT-NIR spectroscopy and SIMCA. Talanta 177, 4-11.

11. Zhang C, Shen T, Liu F, He Y (2018) Identification of coffee varieties using laser-induced breakdown spectroscopy and chemometrics. Sensors 18, ID 95.

12. Mason HY, Lloyd C, Dice M, Sinclair R, Ellis Jr W, Powers L (2003) Taxonomic identification of mi- croorganisms by capture and intrinsic fluorescence detection. Biosens Bioelectron 18, 521-527.

13. Gorbunov MY, Shirsin E, Nikonova E, Fadeev VV, Falkowski PG (2020) A multi-spectral fluorescence induction and relaxation (FIRe) technique for physiological and taxonomic analysis of phytoplankton communities. Mar Ecol Prog Ser 644, 1-13.

14. Tourkya B, Boubellouta T, Dufour E, Leriche F (2009) Fluorescence spectroscopy as a promising tool for a polyphasic approach to pseudomonad taxonomy. Curr Microbiol 58, 39-46.

15. Meyer JM, Geoffroy VA, Baida N, Gardan L, Izard D, Lemanceau P, Palleroni NJ (2002) Siderophore typing, a powerful tool for the identification of fluorescent and nonfluorescent pseudomonads. Appl Environ Microbiol 68, 2745-2753.

16. MacIntyre HL, Lawrenz E, Richardson TL (2010) Taxonomic discrimination of phytoplankton by spectral fluorescence. In: David JS, Ondrej P, Michael AB (eds) Chlorophyll a Fluorescence in Aquatic Sciences: Methods and Applications, Springer, Dordrecht, pp 129-169.

17. Pollastrini M, Holland V, Brüggemann W, Bruelheide H, Dănilă I, Jaroszewicz B, Bussotti F (2016) Taxonomic and ecological relevance of the chlorophyll a fluorescence signature of tree species in mixed European forests. New Phytol 212, 51-65.

18. Aslam I, Afridi MSK (2018) Pharmacognostic characterization of Beaumontia grandiflora (Roxb.) Wall. leaf for taxonomic identification for quality control of a drug. J Appl Res Med Aromat Plants 8, 53-59.

19. Tyystjärvi E, Keränen M, Koski A, Nevalainen O, Aro EM (1998) Chlorophyll fluorescence can be used to identify plant species automatically. In: Shaul M, Wei MZ, Vladimir C, Garab G (eds) Photosynthesis: Mechanisms and Effects, Springer Netherlands, Dordrecht, pp 3857-3860.

20. Talamond P, Verdeil JL, Conéjéro G (2015) Secondary metabolite localization by autofluorescence in living plant cells. Molecules 20, 5024-5037.

21. Pawar HA, Kamat SR (2014) Chemometrics and its application in pharmaceutical field. $J$ Phys Chem Biophys 4, ID 169.

22. Tunnicliffe JM, Cowan T, Shearer J (2015) Chlorogenic acid in whole body and tissue-specific glucose regulation. In: Preedy VR (ed) Coffee in Health and Disease Prevention, Academic Press, UK, pp 777-785.

23. Sousa JS, Neves JCL, Martinez HEP, Alvarez VHV (2018) Relationship between coffee leaf analysis and soil chemical analysis. Rev Bras Cienc Solo 42.

24. Tamimi YN, Silva JA, Yost RS, Hue NV (1997) Adequate nutrient levels in soils and plants in Hawaii (general guide). In: Agronomy and Soils AS3, CTAHR, University of Hawaii at Manoa, Honolulu, USA.

25. Saw AKC, Yam WS, Wong KC, Lai CS (2015) A comparative study of the volatile constituents of south- 
east asian Coffea arabica, Coffea liberica and Coffea robusta green beans and their antioxidant activities. $J$ Essent Oil Bear Plants 18, 64-73.

26. Brand-Williams W, Cuvelier M, Berset C (1995) Use of a free radical method to evaluate antioxidant activity. LWT Food Sci Technol 28, 25-30.

27. Haile M, Bae HM, Kang WH (2020) Comparison of the antioxidant activities and volatile compounds of coffee beans obtained using digestive bio-processing (elephant dung coffee) and commonly known processing methods. Antioxidants 9, ID 408.

28. Phuyal N, Jha PK, Raturi PP, Rajbhandary S (2020) Total phenolic, flavonoid contents, and antioxidant activities of fruit, seed, and bark extracts of Zanthoxylum armatum DC. Sci World $J$ 2020, ID 8780704.

29. Chong J, Wishart DS, Xia J (2019) Using MetaboAnalyst 4.0 for comprehensive and integrative metabolomics data analysis. Curr Protoc Bioinformatics 68, ID 86.

30. Westerhuis JA, Velzen EJ, Hoefsloot HC, Smilde AK (2008) Discriminant Q2 (DQ 2) for improved discrimination in PLSDA models. Metabolomics 4, 293-296.

31. Farrésa M, Platikanova $Y$, Tsakovskib L, Taulera R (2015) Comparison of the variable importance in prediction (VIP) and of the selectivity ratio (SR) variable selection methods in the analysis of three different data sets. $J$ Chemom 29, 528-536.

32. Yisak H, Redi-Abshiro M, Chandravanshi BS (2018) New fluorescence spectroscopic method for the simultaneous determination of alkaloids in aqueous extract of green coffee beans. Chem Centl J 12, 59 .

33. Mubarak A, Croft KD, Bondonno CP, Din NS (2019) Comparison of liberica and arabica coffee: chlorogenic acid, caffeine, total phenolic and DPPH radical scavenging activity. Asian J Agric Biol 7, 130-136.

34. Navarra G, Moschetti M, Guarrasi V, Mangione MR, Leone M (2017) Simultaneous determination of caffeine and chlorogenic acids in green coffee by UV/Vis spectroscopy. J Chem 2017, ID 6435086.

35. Peng DX, Lai F (2012) Using partial least squares in operations management research: A practical guideline and summary of past research. J Oper Manag 30, 467-480.

36. Worley B, Powers R (2013) Multivariate analysis in metabolomics. Curr Metabolomics 1, 92-107.

37. Gaddam RR, Narayan R, Raju KVSN (2016) Fluorescence spectroscopy of nanofillers and their polymer nanocomposites. In: Ponnamma D, Rouxel D, Thomas S (eds) Spectroscopy of Polymer Nanocomposites, Elsevier, Oxford, United Kingdom, pp 158-180. 